\title{
The proteoglycan osteoglycin/mimecan is correlated with arteriogenesis
}

\author{
Andreas Kampmann • Borja Fernández • Elisabeth Deindl · Thomas Kubin • \\ Frederic Pipp · Inka Eitenmüller · Imo E. Hoefer • Wolfgang Schaper • \\ René Zimmermann
}

Received: 12 August 2008/Accepted: 13 October 2008/Published online: 4 November 2008

(C) Springer Science+Business Media, LLC. 2008

\begin{abstract}
Arteriogenesis or collateral growth is able to compensate for the stenosis of major arteries. Using differential display RT-PCR on growing and quiescent collateral arteries in a rabbit femoral artery ligation model, we cloned the rabbit full-length cDNA of osteoglycin/ mimecan. Osteoglycin was present in the adventitia of collateral arteries as a glycosylated protein without keratan sulfate side chains, mainly produced by smooth muscle cells (SMCs) and perivascular fibroblasts. Northern blot, Western blot, and immunohistochemistry confirmed a
\end{abstract}

A. Kampmann · T. Kubin · R. Zimmermann

Research Group Vascular Genomics, Kerckhoff Clinic,

Bad Nauheim, Germany

B. Fernández

Department of Animal Biology, Faculty of Science, University of Málaga, Malaga, Spain

E. Deindl

Walter-Brendel-Center for Experimental Medicine, LMU

Muenchen, Munich, Germany

T. Kubin $\cdot$ R. Zimmermann $(\bowtie)$

Department of Development and Remodeling of the Heart,

Max-Planck-Institute for Heart and Lung Research,

Parkstrasse 1, 61231 Bad Nauheim, Germany

e-mail: rene.zimmermann@mpi-bn.mpg.de

F. Pipp · I. Eitenmüller · W. Schaper

Arteriogenesis Research Group, Max Planck Institute,

Bad Nauheim, Germany

Present Address:

F. Pipp

Institute of Toxicology, Merck KGaA, Darmstadt, Germany

I. E. Hoefer

University Medical Center, Utrecht, The Netherlands collateral artery-specific downregulation of osteoglycin from $6 \mathrm{~h}$ to 3 weeks after the onset of arteriogenesis. Treatment of primary SMCs with the arteriogenic protein fibroblast growth factor-2 (FGF-2) resulted in a similar reduction of osteoglycin expression as observed in vivo. Application of the FGF-2 inhibitor polyanethole sulfonic acid (PAS) blocked the downregulation of osteoglycin and interfered with arteriogenesis. From our study we conclude that downregulation of osteoglycin is a fundamental requirement for proper arteriogenesis.

Keywords Gene expression - Smooth muscle cells · Collateral arteries - Fibroblast growth factor-2 . Proteoglycans · Differential display

\section{Introduction}

Diseases of the cardio-vascular system are the main cause of death in the USA and Europe [1]. Often these problems arise from the occlusion of a main artery, leading to a decrease in blood supply in areas distal to the occlusion. If the stenosis of a large feeding artery proceeds sufficiently slow (e.g., in weeks or months), the human body is able to compensate for the effects of these occlusions, the most effective mechanism of which is the growth of collateral arteries (arteriogenesis) [2]. These collateral arteries develop from preexisting anastomoses creating a natural bypass bridging the occluded artery. The main stimulus for arteriogenesis is shear stress in contrast to angiogenesis which relies on ischemia [3]. In fact, collateral growth is not dependent on ischemia [4, 5]. Arteriogenesis is a complex process, which starts with a structural dilatation of the collateral artery wall, followed by a well-orchestrated mechanism of vascular growth. This requires 
endothelial activation, leukocyte recruitment, cell proliferation, cell death, smooth muscle cell (SMC) phenotypic shift, and vascular matrix remodeling [6]. The whole process is triggered by various growth factors and cytokines, e.g., monocyte chemoattractant protein (MCP-1) or fibroblast growth factor-2 (FGF-2). These factors stimulate arteriogenesis in a rabbit model, leading to a faster compensation of the blood flow deficit caused by the occlusion of the femoral artery [7, 8]. In addition, genes which are involved in the regulation of the cytoskeleton or the extracellular matrix (ECM) or others like Carp (cardiac ankyrin repeat protein) [9] and urokinase plasminogen activator [4] are differentially expressed during arteriogenesis.

In order to identify additional genes involved in collateral growth, we started a screen for differentially expressed genes in rabbit collateral arteries after 3 days and 3 weeks of ligation of the femoral artery using differential display reverse transcription-polymerase chain reaction (DDRTPCR) [10].

One of the genes identified was the rabbit orthologue ${ }^{1}$ of human osteoinductive factor/osteoglycin [11] or mimecan [12]. Osteoglycin belongs to the family of small leucine-rich proteoglycans, a group of currently 11 proteins, characterized by leucine-rich repeats in their central domain. These proteins are located in the ECM and are important for the regulation of the structure of the matrix, but also in the regulation of cell cycle and growth factor actions [13-15]. Here, we present an in depth study on osteoglycin in a rabbit model of arteriogenesis. We cloned the full-length cDNA of rabbit osteoglycin and investigated its expression on RNA and protein level in growing collateral arteries, in a time course of 3 weeks starting with the induction of arteriogenesis by femoral artery ligation. The distribution of osteoglycin mRNA and protein was explored by in situ hybridization and immunohistochemistry, respectively. To define the modification status of the protein, we performed Western blot analysis on $\mathrm{N}$-glycanase treated osteoglycin. In addition, we carried out several in vitro studies using factors known to stimulate either arteriogenesis or SMC proliferation, aiming to characterize their potential to regulate osteoglycin expression. Finally, we used the rabbit femoral ligation model to test the capacity of one of these factors (FGF-2) to regulate osteoglycin expression in vivo and to confirm the relevance of this proteoglycan in the arteriogenic process.

\footnotetext{
1 The nucleotide sequence reported in this article has been submitted to the GenBank Data Bank with accession number AF487889.
}

\section{Material and methods}

Animal model

To induce collateral artery growth, we used the femoral artery ligation model in New Zealand White rabbits as described before [16]. In brief, rabbits were anesthetized with a ketamine/xylacine mixture and the right femoral artery was occluded with two ligatures $1.5 \mathrm{~cm}$ apart from each other. As a control the left femoral artery was sham operated. Animal care and all experimental procedures were performed in accordance to the German and National Institutes of Health animal legislation guidelines and were approved by the local animal care and use committees.

For gene expression studies on RNA and protein level, collateral arteries were isolated 3, 6, 12, $24 \mathrm{~h}, 3,7$ days, and 3 weeks after occlusion of the femoral artery or after sham operation. All samples were snap frozen in liquid nitrogen or embedded in Tissue-teck mounting media (Sakura Finitek, Heppenheim, Germany) and stored at $-80^{\circ} \mathrm{C}$ until further analyses. Postmortem angiograms of the whole hind limb were performed as previously described [16].

\section{Infusion of PAS}

The experimental treatment and the surgical procedure were performed as previously described [17]. In brief, after ligation of the right femoral artery, rabbits (body weight $3.7 \pm 0.5 \mathrm{~kg}$, aged 3-4 months; $n=4)$ received polyanethole sulfonic acid (PAS) $(15 \mathrm{mg} / \mathrm{kg}$ per day, Peprotech, Frankfurt, Germany), via an osmotic minipump connected to the collateral circulation. All animals tolerated the treatment well. At day 7, rabbits were subjected to angiography, and collateral arteries from the occluded and the control legs were isolated and snap frozen as described above.

RNA isolation, DDRT-PCR, and Northern blot

Isolation of total RNA, DDRT-PCR, and Northern blot were performed according to standard procedures [18-20]. Differentially expressed cDNAs were cloned in pGEM-T Easy vector (Promega, Mannheim, Germany) or in pCRScript Amp SK(+) (Stratagene, La Jolla, USA) and sequenced. Full-length osteoglycin cDNA was obtained by 5' RACE using a SMART RACE cDNA amplification kit (Clontech, Mountain View, USA). For Northern hybridization, $15 \mu \mathrm{g}$ total RNA from growing and quiescent collateral arteries were separated on a $1 \%$ agarose gel containing 0.66 M formaldehyde and transferred to Nylon membrane (Duralon UV-membrane, Stratagene, La Jolla, USA). The blots were hybridized with [32P] dCTP 
random-primed labeled cDNA probes (Rediprime II, Amersham Biosciences, Freiburg, Germany), exposed to Storage phosphor screens overnight, and scanned using a Storm 860 PhosphorImager (Amersham Biosciences, Freiburg, Germany). For normalization, blots were rehybridized with a specific 18S RNA oligonucleotide as described previously [4]. Quantification of the signals was done using ImageQuant software (Amersham Biosciences, Feiburg, Germany). All experiments were repeated three times independently.

\section{Antibodies and Western blot analyses}

Tissue samples from growing and quiescent collateral arteries were disrupted in extraction buffer $(0.1 \mathrm{M}$ Tris$\mathrm{HCl}, 0.01 \mathrm{M}$ EDTA, $0.04 \mathrm{M}$ DTT, 10\% SDS, pH 8.0) using an ultrasonic device (Sonoplus G70, Bandelin, Berlin, Germany) with the following settings: five times $30 \mathrm{~s}$, $40 \%$ power, $50 \%$ cycle. Proteins lysates were separated on a 4-12\% Bis-Tris gel (Invitrogen, Karlsruhe, Germany). To deglycosylate protein samples, $50 \mu \mathrm{g}$ of protein was denatured at $100^{\circ} \mathrm{C}$ for $10 \mathrm{~min}$ in $1 \times$ Denaturing buffer (NEB, Frankfurt, Germany), in a total volume of $12 \mu \mathrm{l}$. The denatured protein mixture was placed on ice, and $2 \mu \mathrm{l}$ $10 \times$ G7 buffer, $2 \mu \mathrm{l} 10 \% \mathrm{NP}-40$, and $2 \mu \mathrm{l}$ PNGase F (500 U/ $\mu 1$, NEB, Frankfurt, Germany) were added. The reaction continued for $2 \mathrm{~h}$ at $37^{\circ} \mathrm{C}$. Deglycosylated proteins were separated on a $4-12 \%$ Bis-Tris gel (Invitrogen, Karlsruhe, Germany).

Osteoglycin was detected on the blots using a rat polyclonal antibody produced by Eurogentec (Double XP program, Koeln, Germany) after immunization with a peptide derived from rabbit osteoglycin (amino acids 79-84, VTPAPPKKENDEMPTC). The secondary antibody was a peroxidase-conjugated goat anti-rat IgG (Santa Cruz, Heidelberg, Germany). Immunoreactive bands were visualized with the ECL system (Amersham Biosciences, Freiburg, Germany). All experiments were repeated independently three times.

In situ hybridization and immunohistochemistry

The analyses of osteoglycin mRNA and protein in the growing collaterals by in situ hybridization and immunochemistry were done as described recently [21].

Furthermore, when appropriate, in situ hybridization with osteoglycin RNA probes or immunoperoxidase staining with the protein was combined with immunofluorescence with vascular smooth muscle $\alpha$-actin antibodies (Sigma, Munich, Germany; data not shown) or with the proliferation marker ki67 (Dako, Hamburg, Germany) in consecutive cryosections [21]. The omission of the first antibody served as negative control.
Immunohistochemistry and photomicrography for osteoglycin were performed equally in all the sections, in order to minimize variability generated by the method. The intensity of osteoglycin immunostaining was qualitatively compared between sections, taken at comparable locations, from quadriceps muscles of six occluded and six sham operated rabbits.

\section{Cell culture}

We used primary cultures of rabbit aortic SMCs, which were isolated as described previously for porcine aortic SMCs [22]. Cells were routinely cultured in medium 199 (PAA, Coelbe, Germany) with 20\% FCS and splitted 1:4 upon confluence. For stimulation, cells between passages 4 and 6 were serum starved for 3 days. Stimulation was done with Oncostatin M (OsM, $10 \mathrm{ng} / \mathrm{ml})$, FGF-2 $(20 \mathrm{ng} / \mathrm{ml})$, transforming growth factor- $\beta$ (TGF- $\beta_{1}, 5 \mathrm{ng} / \mathrm{ml}$ ), platelet derived growth factor-AB (PDGF-AB, $10 \mathrm{ng} / \mathrm{ml}$ ), and granulocyte-macrophage colony-stimulating factor (GMCSF, $10 \mathrm{ng} / \mathrm{ml}$ ). All factors used were recombinant human (Cell Concepts, Umkirch, Germany).

To test the influence of MCP-1 (10 ng/ml, Boehringer, Mannheim, Germany) on growth factor actions, all stimulations were repeated as co-stimulations with this chemokine. RNA was isolated from stimulated and costimulated cells after 24,48 , and $72 \mathrm{~h}$, with peqGOLD RNApure (PEQLAB, Erlangen, Germany). All experiments were repeated three times.

\section{Statistics}

Statistical comparisons were performed with ANOVA and Bonferroni tests. $P$ values $<0.05$ were regarded as statistical significant.

\section{Results}

Cloning and characterization of the rabbit osteoglycin cDNA

Using DDRT-PCR, we identified 191 differentially expressed genes, most of which were downregulated at $3 \mathrm{~d}$ and 3 weeks. One of these genes showed a strong homology $(77 \%)$ to the bovine osteoinductive factor (accession no. M37974). We cloned the full-length cDNA of $2.5 \mathrm{~kb}$ by $5^{\prime}$ RACE and found that the gene represented the rabbit orthologue of the bovine osteoglycin/mimecan (Fig. 1). A single open reading frame of $896 \mathrm{bp}$ coded for a protein of 298 amino acids. Conserved amino acid elements included an $\mathrm{N}$-terminal signal peptide and three consensus sequences for N-linked glycosylation. Furthermore, the amino 


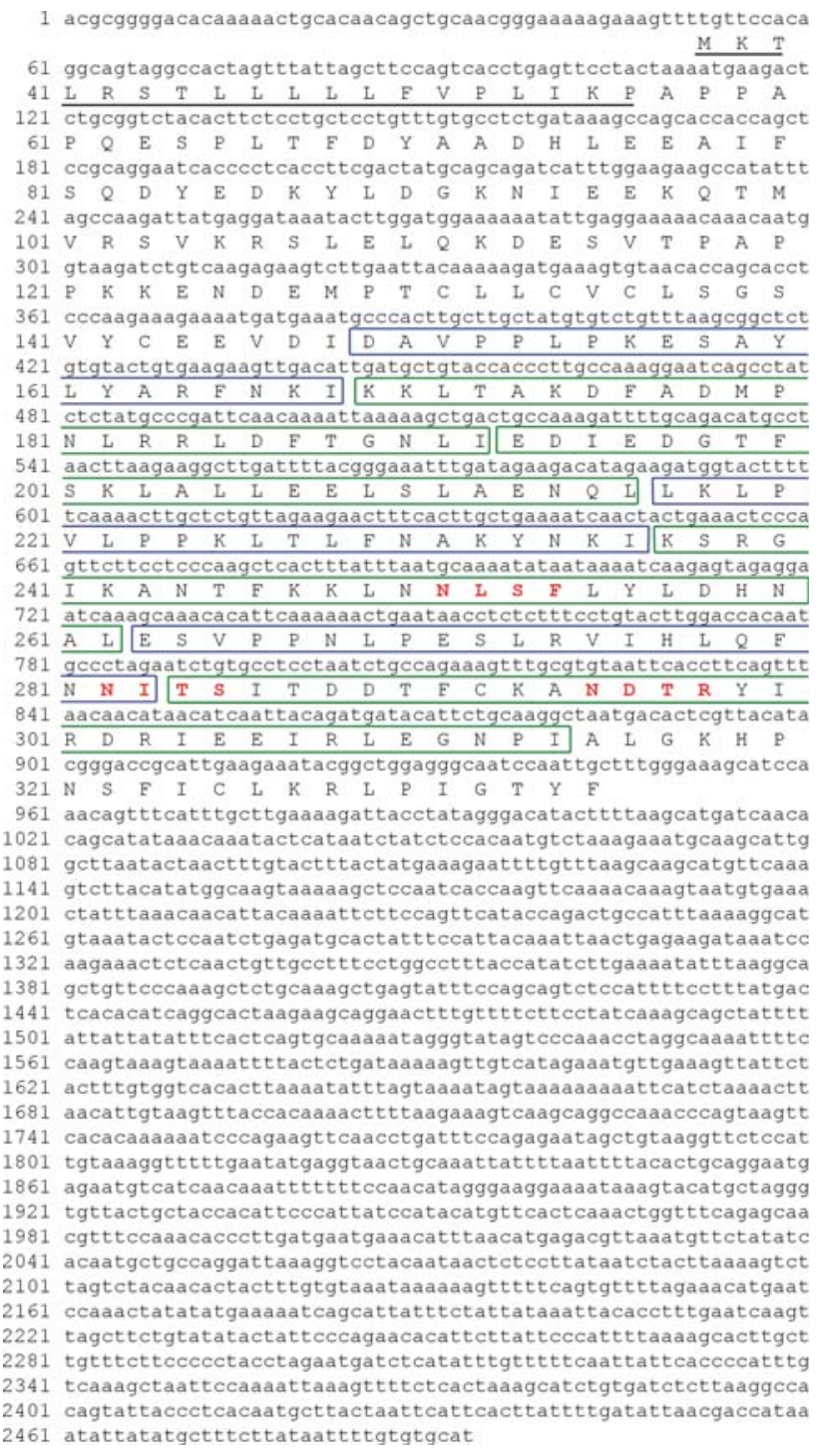

Fig. 1 Sequence of rabbit osteoglycin. The rabbit osteoglycin cDNA consisted of 2,493 bp and contained an open reading frame coding for a protein of $298 \mathrm{aa}$. The protein starts with a signal peptide of 19 amino acids (underlined). The three consensus sequences for $\mathrm{N}$-linked glycosylation are printed in red. The leucine-rich repeats of the S-type are marked in blue, that of the T-type in green

acid sequence showed seven leucine rich repeats; three of them represented the 21 amino acid $\mathrm{S}$ type and four of them the longer 26 amino acid T type (Fig. 1). The comparison of the rabbit osteoglycin amino acid sequence to the human, bovine, and rat ones showed an identity of $88 \%$, $87 \%$, and $83 \%$, respectively (data not shown).

\section{Osteoglycin expression in collateral arteries}

Northern blot analyses on rabbit collateral arteries showed a $3.2 \mathrm{~kb}$ mRNA which was downregulated at nearly all time points studied (Fig. 2). The mRNA level was

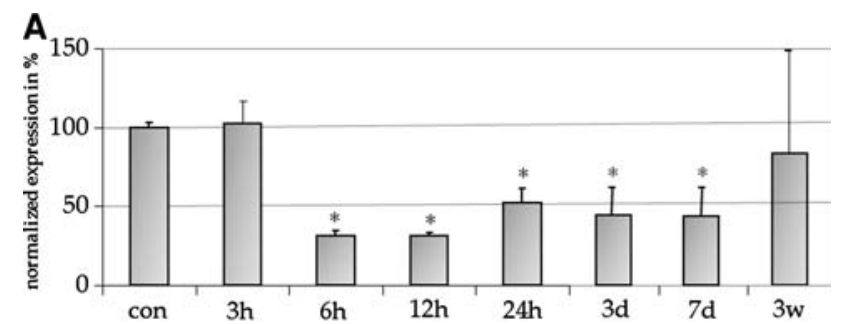

B

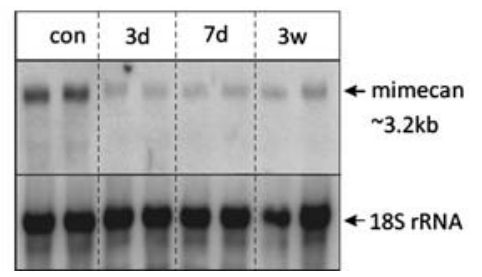

Fig. 2 Osteoglycin mRNA expression at various time points after occlusion of the femoral artery. A Bar graphs representing Northern blot results on osteoglycin expression in growing collateral arteries from $3 \mathrm{~h}$ to 3 weeks after femoral artery ligation (for 3-24 h: $n=4$ for each time point; for $3 \mathrm{~d}$ to $3 \mathrm{w}: n=8$ for each time point; control: $n=8)$. B Representative Northern blot showing osteoglycin expression (top) in quiescent (con) and growing collateral arteries at days 3, 7 as well as 3 weeks after femoral artery occlusion. The blot was rehybridized with an $18 \mathrm{~S}$ rRNA specific probe (bottom). Results are expressed as mean \pm SEM $(* P<05$ versus con; con control)

decreased to $31 \%$ of the control value as early as 6 and $12 \mathrm{~h}$ after femoral artery ligation (all values $P<0.05$, Fig. 2A). One day after surgery, osteoglycin production was still $52 \%$ of the control value, remaining at low levels during the entire first week of arteriogenesis ( 3 days, $43 \%$ and 7 days, 42\%, of control; $P<0.05$; Fig. 2B). After 3 weeks, osteoglycin expression started to return to control levels ( $86 \%$ of control; not significant).

Western blot analyses revealed a double band of approximately $36-40 \mathrm{kDa}$ (Fig. 3A), corresponding to glycosylated osteoglycin (see below). Changes in the expression level of the protein during arteriogenesis were comparable to those at the mRNA level. The protein content was reduced to $43 \%$ of the control value $(P<0.001)$ after 3 days and to $48 \%(P<0.001)$ after 7 days of surgery (Fig. 3A, B). In contrast to RNA, the protein level was still low 3 weeks after occlusion of the femoral artery $(40 \%$ of control value; $P<0.01$ ).

Osteoglycin was initially described as a keratan sulfate proteoglycan. The core protein can be modified with oligosaccharides at three possible sites for $\mathrm{N}$-glycosylation present in the osteoglycin sequence (Fig. 1). To test whether the detected signals of immunoreactive osteoglycin reflected the unmodified or the glycosylated protein in collateral arteries, the oligosaccharide side chains were removed with $\mathrm{N}$-glycanase, resulting in a shift in the size of the protein of approximately $3 \mathrm{kDa}$ (Fig. 3C), indicating that osteoglycin is expressed in collateral arteries as a glycoprotein without keratan sulfate side chains. 

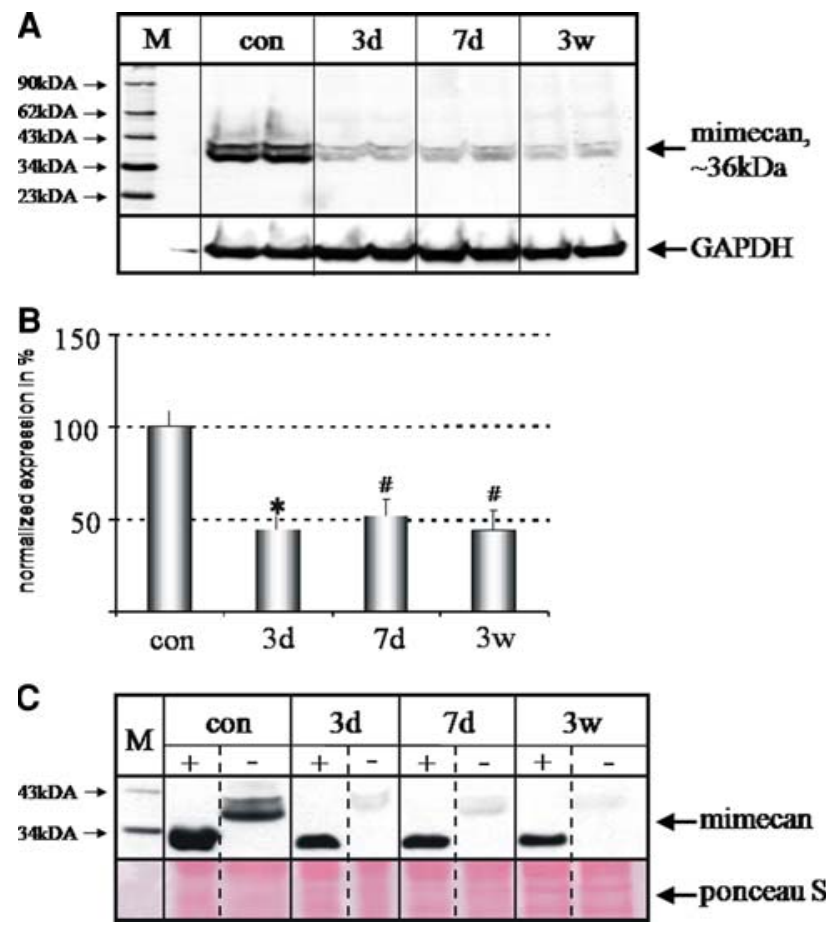

Fig. 3 Western blot analyses of osteoglycin. A Representative Western blot showing the level of osteoglycin protein in growing collateral arteries at days 3, 7 and 3 weeks after femoral artery occlusion as well as in control vessels. B Quantification of the Western blot analyses. $n=7$ for control and for $\mathrm{d} 3, n=6$ for $\mathrm{d} 7$, and $n=5$ for 3 weeks. Results are expressed as mean \pm SEM $(* P<05$ versus con). C Protein preparations before $(-)$ and after $(+)$ deglycosylation ( $n=4$ for each time point). Ponceau $S$ staining was used in all Western blots to demonstrate equal loading of the protein samples. ( $M$ Cruz marker molecular weight standard, con control, $3 d 3$ days after occlusion, $7 d 7$ days after occlusion, $3 w$ 3 weeks after occlusion)

Localization of osteoglycin mRNA and protein

The sites of osteoglycin production and accumulation in collateral arteries were investigated in detail by in situ hybridization (Fig. 4A, C, D) and immunohistochemistry (Fig. 4B, E, F), respectively. In collateral arteries, osteoglycin mRNA was mainly expressed by SMCs of the media and cells of the adventitia (Fig. 4C, D). In perivascular areas, signals were prominent in the fibroblasts of the fascia that envelops the skeletal muscles. Localization of osteoglycin protein was analyzed using the same antibody as for Western blot (Fig. 4E). The protein was accumulated in the ECM, with the strongest signals present in the adventitia of the arteries and the external fascia of the quadriceps muscles. Moderate signals were detected in the matrix surrounding individual muscle fibers. After 7 days of collateral artery growth, the amount of osteoglycin protein was clearly diminished in the adventitia of growing collateral arteries (Fig. 4F). These were distinguishable from neighboring quiescent arteries by the high percentage of proliferating SMCs in the media (Fig. 4G). Only growing collateral arteries from ligated animals showed a reduction of osteoglycin signals in the adventitia, whereas quiescent arteries from the same individuals, and from sham operated animals, as well as the muscle fascia of every specimen showed a constant intensity of staining (compare Fig. 4E, F).

Factors influencing osteoglycin expression in vitro

Primary rabbit aortic SMCs were used to investigate the influence of growth factors on the expression of osteoglycin (Fig. 5). While most factors used showed no or only a negligible effect Oncostatin $\mathrm{M}$ led to a significant reduction of osteoglycin mRNA to $40 \%$ of the control value after $24 \mathrm{~h}$ of stimulation, $31 \%$ after $48 \mathrm{~h}(P<0.05)$, and $27 \%$ after $72 \mathrm{~h}(P<0.05)$. FGF-2 showed a comparable but biphasic effect, with a downregulation to $36 \%$ of control value after $24 \mathrm{~h}(P<0.05)$, expression levels of $80 \%$ after $48 \mathrm{~h}$, dropping again to $40 \%$ after $72 \mathrm{~h}$. TGF- $\beta_{1}$ had only a short, limited effect after $48 \mathrm{~h}$ of stimulation, similar to PDGF-AB, with a downregulation to $60 \%$ and $63 \%$ of the control value, respectively. GM-CSF or MCP-1 alone showed no substantial effect.

MCP-1 is a well-known stimulator of arteriogenesis when used either isolated or in combination with other factors like GM-CSF. Co-stimulation of TGF- $\beta_{1}$ with MCP-1 caused a deeper effect on osteoglycin downregulation (24 h: 89\%, $48 \mathrm{~h}: 40 \%, 72 \mathrm{~h}: 29 \%)$ than single TGF$\beta_{1}$ stimulation (Fig. 5). Co-stimulations using GM-CSF and MCP- 1 or GM-CSF, TGF- $\beta_{1}$, and MCP- 1 also showed prominent but non-significant reductions when compared to single stimulations.

FGF-2 as a modulator of osteoglycin expression in vivo

As shown above, FGF-2, a key factor for arteriogenesis, induced a pattern of osteoglycin downregulation in SMCs similar to the pattern observed in vivo. Thus, we decided to investigate whether FGF-2 plays a role in the regulation of osteoglycin expression in vivo. Surgical occlusion of the femoral artery was accompanied with continuous systemic infusion of the FGF-2 inhibitor PAS during 7 days. Postmortem angiograms of PAS treated animals showed a strong reduction in collateral growth after femoral ligation when compared to untreated rabbits (Fig. 6A) who had big collateral arteries with a corkscrew-like course (Fig. 6C). In contrast, PAS treated rabbits showed small collateral arteries after femoral surgery, with no corkscrew formation (Fig. 6B).

In contrast to untreated rabbits (Fig. 2), PAS treated specimens had no significant change of osteoglycin mRNA expression after 7 days of femoral artery ligation (Fig. 6D). 
Fig. 4 Localization of osteoglycin mRNA (A, C, D), protein $(\mathbf{B}, \mathbf{E}, \mathbf{F})$, and the proliferation marker ki-67 (G) in collateral arteries and surrounding tissue. A, C, D In situ hybridization. A The hybridization with the sense probe was used as a negative control. C, D Positive signals for osteoglycin were detected in the SMCs of the collateral media (arrows) and in fibroblasts (arrowheads). B, E, F Immunohistochemistry. B Negative control for immunoperoxidase reaction. $\mathbf{E}$ Quiescent collateral arteries showed a thin media and continuous internal elastic lamina (arrow). Note the prominent staining for osteoglycin in the adventitia (black arrowheads). F, G (consecutive sections): After 7 days of femoral artery occlusion growing collateral arteries show fragmented elastic lamina (arrows in F), prominent neointima formation (NI in $\mathbf{F}$ ), and SMC proliferation (arrows in $\mathbf{G}$; ki67 staining). Note the strong reduction of osteoglycin protein in the adventitia of growing collateral arteries (compare black arrowheads in $\mathbf{E}$ and $\mathbf{F}$ ), whereas in the fascia of the muscle the signal intensity remains the same (red arrowheads)

Fig. 5 Stimulation of SMCs with distinct cytokines and growth factors. Bar graphs representing Northern blot results on osteoglycin expression in primary rabbit SMCs after 24, 48, and $72 \mathrm{~h}$ of stimulation with distinct growth factors and cytokines. The factors and concentrations used are indicated in the "Material and Methods" section. Results are expressed as mean \pm SEM (* $P<0.05$ versus con; con control, OsM Oncostatin M). All experiments were performed in triplicate
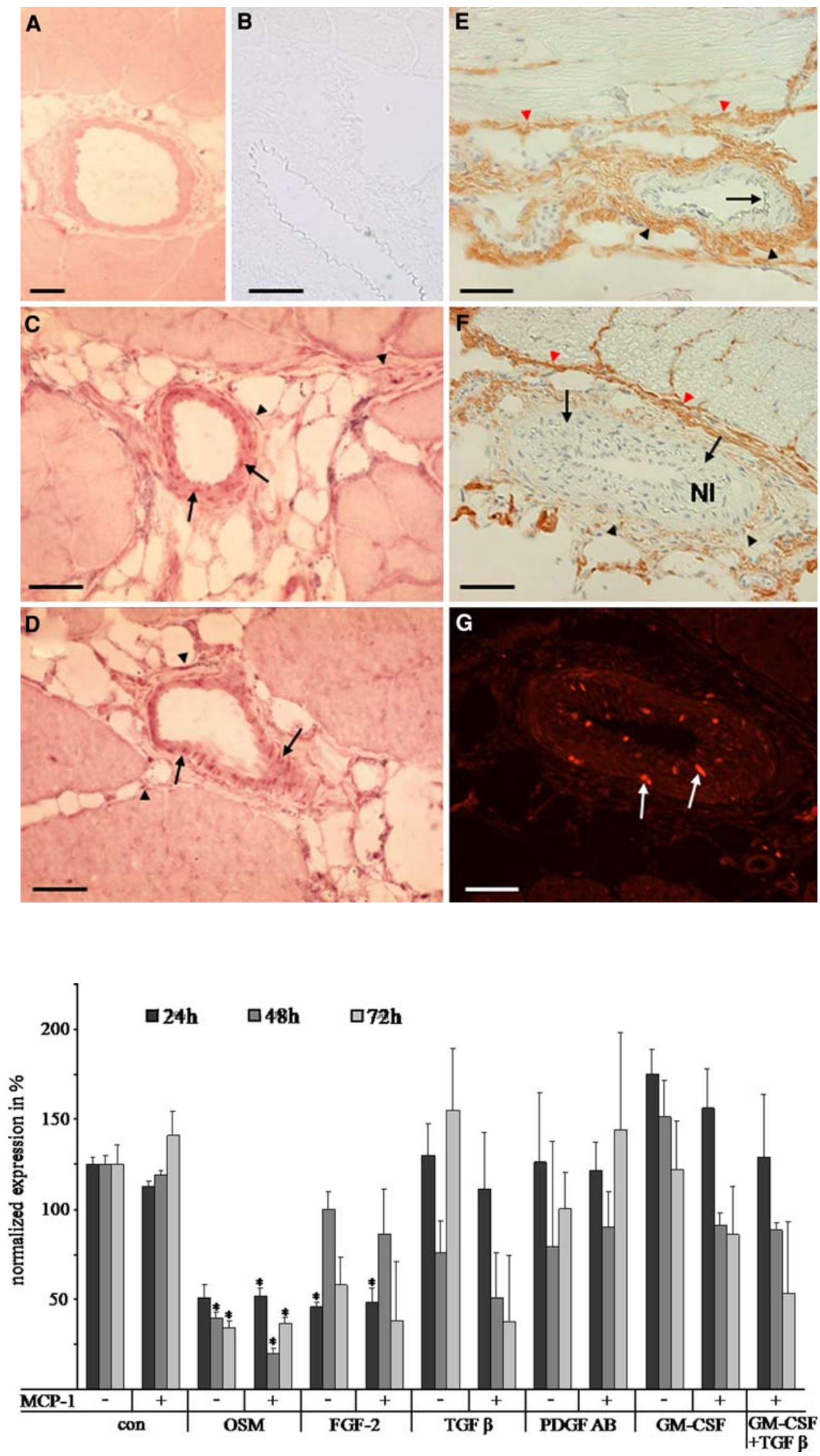
Fig. 6 Application of PAS. Postmortem angiograms of rabbit hind limbs. A After 1 week of infusion of PAS without ligation of the femoral artery. B After 1 week of femoral artery ligation with concomitant infusion of PAS. C After 1 week of femoral artery ligation without infusion of PAS. Upon femoral artery ligation, only rabbits not treated with PAS (C) showed collateral arteries with corkscrew formation (arrows), a typical sign for growing collaterals. D Bar graphs representing Northern blot results on osteoglycin expression after 1 week of infusion of PAS in resting collateral arteries (con + PAS) and in growing collateral arteries 7 days after ligation of the femoral artery $(7 \mathrm{~d}$ occ $+\mathrm{PAS} ; n=4)$. Results are expressed as mean \pm SEM. No statistical difference was found

Thus, PAS treatment abolished osteoglycin downregulation in collateral arteries induced by femoral ligation.

\section{Discussion}

Our study defines rabbit osteoglycin, a protein of 298 amino acids and a prototype member of the family of small leucine-rich proteins (SLRP; for an overview see [14]) as a critical component of arteriogenesis. Northern blot results showed a single band of $3.2 \mathrm{~kb}$ for rabbit osteoglycin mRNA, which is in agreement with the published mRNA size for rat [23], therefore excluding differential splicing or alternative polyadenylation. The mRNA is translated in a glycosylated protein without keratan sulfate side chains of $36-40 \mathrm{kDa}$ fitting with the theoretical mass (including the signal peptide) of $34 \mathrm{kDa}$ [23].

The comparative study of osteoglycin expression in collateral arteries of rabbits subjected to femoral artery occlusion indicated a clear downregulation during the entire period under investigation. Localization of osteoglycin mRNA and protein in skeletal muscles and vessels corresponded to that shown in previous studies [21]. One week after induction of arteriogenesis, osteoglycin protein was clearly diminished in the adventitia of growing collateral arteries, whereas the surrounding muscle fascia and quiescent vessels were unaffected. These data also illustrate the specificity of osteoglycin function in the process, as changes in the amount of the protein were restricted to growing collateral arteries, whereas osteoglycin amount in the skeletal muscle matrix did not change.

Our results strongly support previous reports showing that osteoglycin expression is linked to SMC proliferation [23] because osteoglycin downregulation during arteriogenesis coincides with the proliferation phase of SMCs. In the rabbit hind limb model, proliferation starts $24 \mathrm{~h}$ after femoral artery occlusion, peaks between days 2 and 3 , and gradually decreases until day 21 [24]. Moreover, downregulation starts even before mitosis is detected $(6$ and $12 \mathrm{~h}$ after surgery), suggesting that osteoglycin may be involved in SMC activation.
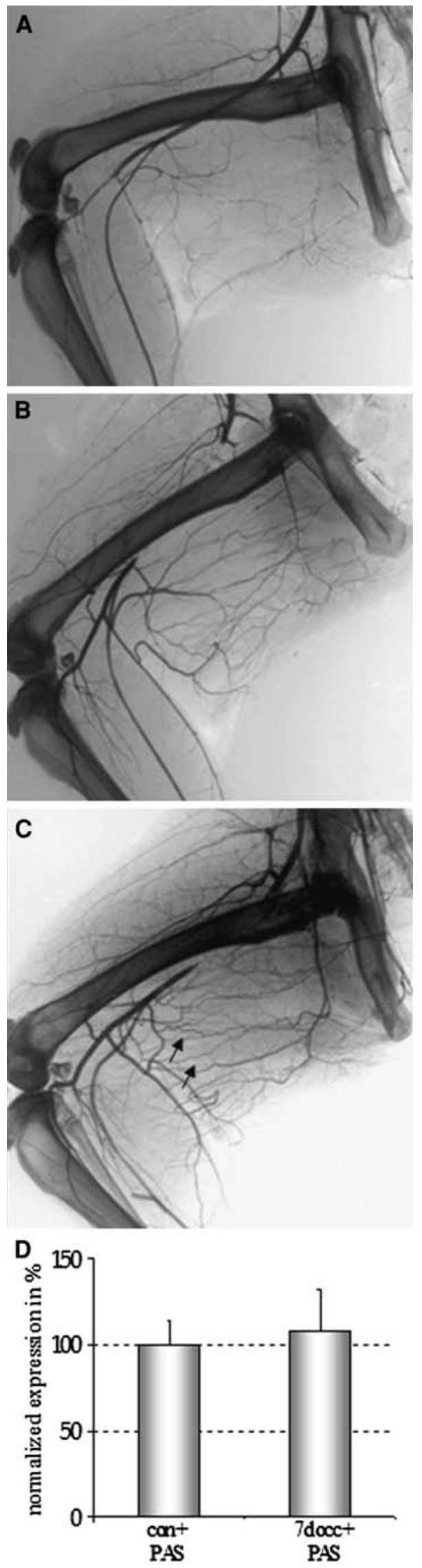
We found evidence that application of FGF-2, a potent mitogen for SMCs and fibroblasts and a critical element in arteriogenesis [17], to SMCs in vitro closely mimicked the downregulation of osteoglycin observed during the first 3 days of arteriogenesis in vivo, similar to the one previously observed in bovine keratocytes [25] and human SMCs [23].

In addition, infusion of PAS, a nontoxic sulfonic acid polymer described to block the action of FGF-2 via complex formation [26] and strongly interfering with arteriogenesis in vivo [17], completely inhibited the downregulation of osteoglycin. This demonstrates that FGF-2 is either directly or indirectly involved in regulating the expression of osteoglycin during the process of arteriogenesis in vivo, probably via the high-affinity receptor FGFR-1 which is expressed by SMCs of the collateral wall, whereas FGF-2 is mostly secreted by recruited macrophages [17]. Macrophage invasion and activation in the adventitia of collateral arteries by secreted cytokines like MCP-1 are critical events during arteriogenesis [27-29]. Interestingly, in our SMC cultures, MCP-1 co-stimulation enhanced the capacity of most factors tested to downregulate osteoglycin. Furthermore, Oncostatin M, another macrophage-secreted cytokine with a known mitogenic effect on SMCs [30, 31], induced a significant downregulation of osteoglycin at every time point studied. These data indicate that factors other than FGF-2 may influence osteoglycin expression during collateral growth and support the hypothesis that osteoglycin downregulation is a necessary mechanism for arteriogenesis progression.

The results of the present study point to a function of osteoglycin as a negative regulator of the mitotic activity in the wall of collateral arteries. However, the main function of osteoglycin as with other SLRPs may be the regulation of collagen fibrillogenesis in the ECM [14, 32]. Osteoglycin knockout mice show reduced tensile strength of the skin, caused by alterations in the diameters of collagen fibrils, thereby supporting a role for this proteoglycan in determining elasticity and tensile strength [21, 32]. During rabbit peripheral arteriogenesis, collateral artery remodeling leads to a final increase in size of 10-fold the original vessel diameter [7]. It is reasonable to propose that osteoglycin downregulation may influence the structure of the collagen matrix during the remodeling phase of arteriogenesis, allowing the structural dilatation of collateral arteries. New studies with osteoglycin knockout mouse models may help in understanding how this glycoprotein influences the mechanical properties of the vascular wall during vascular disease and adaptative growth.

Finally, studies have shown that osteoglycin binds bone morphogenetic proteins (BMPs), members of the TGF- $\beta$ superfamily, thereby regulating their bioavailability and action [33]. Additional studies are required to address the possible involvement of BMPs in arteriogenesis and their regulation by osteoglycin.

Acknowledgment We thank Prof. Dr. Dr. Thomas Braun for his support and the allowance to finish this work in his department. The authors appreciate the technical assistance of Marianne Granz, Kerstin Richter, and Claudia Ullmann and the expertise of Frank Voss and Gerd Stämmler in performing statistical analysis.

\section{References}

1. WHO (1999) WHO Library Cataloguing in Publication Data. The world health report 1999: Making a difference I. Title: Making a difference ISBN 9241561947 (NLM Classification: WA 540.1) ISSN 1020-3311

2. Schaper W, Piek JJ, Munoz-Chapuli R et al (1999) Collateral circulation of the heart. In: Ware JA, Simons M (eds) Angiogenesis and cardiovascular disease. Oxford University Press, New York, pp 159-198

3. Eitenmuller I, Volger O, Kluge A et al (2006) The range of adaptation by collateral vessels after femoral artery occlusion. Circ Res 99:656-662. doi:10.1161/01.RES.0000242560.77512.dd

4. Deindl E, Ziegelhoffer T, Kanse SM et al (2003) Receptorindependent role of the urokinase-type plasminogen activator during arteriogenesis. FASEB J 17:1174-1176

5. Ito WD, Arras M, Winkler B et al (1997) Angiogenesis but not collateral growth is associated with ischemia after femoral artery occlusion. Am J Physiol 273:H1255-H1265

6. Arras M, Ito WD, Scholz D et al (1997) Monocyte activation in angiogenesis and collateral growth in the rabbit hindlimb. J Clin Invest 101:40-50. doi:10.1172/JCI119877

7. Hoefer IE, van Royen N, Buschmann IR et al (2001) Time course of arteriogenesis following femoral artery occlusion in the rabbit. Cardiovasc Res 49:609-617. doi:10.1016/S0008-6363 (00)00243-1

8. Unger EF, Banai S, Shou M et al (1994) Basic fibroblast growth factor enhances myocardial collateral flow in a canine model. Am J Physiol 266:H1588-H1595

9. Boengler K, Pipp F, Fernandez B et al (2003) Arteriogenesis is associated with an induction of the cardiac ankyrin repeat protein (carp). Cardiovasc Res 59:573-581. doi:10.1016/S0008-6363(03) 00511-X

10. Kampmann A, Fernandez B, Kubin T et al (2001) Osteoglycin: a potential new target for the promotion of arteriogenesis? Circulation 104:11-235

11. Bentz H, Nathan R, Rosen D et al (1989) Purification and characterization of a unique osteoinductive factor from bovine bone. J Biol Chem 264:20805-20810

12. Funderburgh JL, Corpuz LM, Roth MR et al (1997) Mimecan, the $25-\mathrm{kDa}$ corneal keratan sulfate proteoglycan, is a product of the gene producing osteoglycin. J Biol Chem 272:28089-28095. doi: $10.1074 /$ jbc. 272.44 .28089

13. Csordas G, Santra M, Reed CC et al (2000) Sustained downregulation of the epidermal growth factor receptor by decorin. A mechanism for controlling tumor growth in vivo. J Biol Chem 275:32879-32887. doi:10.1074/jbc.M005609200

14. Iozzo R (1997) The family of small leucine-rich proteoglycans: key regulators of matrix assembly and cellular growth. Crit Rev Biochem Mol Biol 32:141-174. doi:10.3109/1040923970910 8551

15. Zimmermann R, Kampmann A, Kubin T et al (2004) Differential expression of the extracellular matrix (ECM) components mimecan and elastin during arteriogenesis. J Mol Cell Cardiol 37:170 
16. Ito WD, Arras M, Winkler B et al (1997) Monocyte chemotactic protein-1 increases collateral and peripheral conductance after femoral artery occlusion. Circ Res 80:829-837

17. Deindl E, Hoefer IE, Fernandez B et al (2003) Involvement of the fibroblast growth factor system in adaptive and chemokineinduced arteriogenesis. Circ Res 92:561-568. doi:10.1161/ 01.RES.0000061181.80065.7D

18. Chomczynski P, Sacchi N (1987) Single step method of RNA isolation by acid guanidinium thiocyanate-phenol-chloroform extraction. Anal Biochem 162:156-159. doi:10.1016/0003-2697 (87)90021-2

19. Liang P, Pardee AP (1992) Differential display of eukaryotic messenger RNA by means of the polymerase chain reaction. Science 257:967-971. doi:10.1126/science.1354393

20. Sambrook J, Fritsch EF, Maniatis T (1989) Molecular cloning: a laboratory manual. Cold Spring Harbor Laboratory Press, Cold Spring Harbor

21. Fernandez B, Kampmann A, Pipp F et al (2003) Osteoglycin expression and localization in rabbit tissues and atherosclerotic plaques. Mol Cell Biochem 246:3-11. doi:10.1023/A:10234874 24751

22. Kubin T, Vogel S, Wetzel J et al (2003) Porcine aortic endothelial cells show little effects on smooth muscle cells but are potent stimulators of cardiomyocyte growth. Mol Cell Biochem 242:3945. doi:10.1023/A:1021177326151

23. Shanahan CM, Cary NRB, Osbourn JK et al (1997) Identification of osteoglycin as a component of the vascular matrix. Differential expression during neointima formation and in atherosclerotic plaques. Arter Thromb Vasc Biol 17:2437-2447

24. Scholz D, Ito W, Fleming I et al (2000) Ultrastructure and molecular histology of rabbit hind-limb collateral artery growth (arteriogenesis). Virchows Arch 436:257-270. doi:10.1007/ s004280050039
25. Long CJ, Roth MR, Tasheva ES et al (2000) Fibroblast growth factor-2 promotes keratan sulfate proteoglycan expression by keratocytes in vitro. J Biol Chem 275:13918-13923. doi:10.1074/ jbc.275.18.13918

26. Liekens S, Neyts J, Degréve B et al (1997) The sulfonic acid polymers PAMPS [poly(2-acrylamido-2-methyl-1-propanesulfonoc acid)] and related analogons are highly potent inhibitors of angiogenesis. Oncol Res 9:173-181

27. Bergmann CE, Hoefer IE, Meder B et al (2006) Arteriogenesis depends on circulating monocytes and macrophage accumulation and is severely depressed in op/op mice. J Leukoc Biol 80:59-65. doi:10.1189/jlb.0206087

28. Ito B, Tate H, Kobayashi M et al (1987) Reversibly injured, postischemic canine myocardium retains normal contractile reserve. Circ Res 61:834-846

29. Voskuil M, van Royen N, Hoefer IE et al (2003) Modulation of collateral artery growth in a porcine hindlimb ligation model using MCP-1. Am J Physiol Heart Circ Physiol 284:H1422H1428

30. Grove RI, Eberhardt C, Abid S et al (1993) Oncostatin M is a mitogen for rabbit vascular smooth muscle cells. Proc Natl Acad Sci USA 90:823-827. doi:10.1073/pnas.90.3.823

31. Rose TM, Bruce AG (1991) Oncostatin M is a member of a cytokine family that includes leukemia-inhibitory factor, granulocyte colony-stimulating factor, and interleukin 6. Proc Natl Acad Sci USA 88:8641-8645. doi:10.1073/pnas.88.19.8641

32. Tasheva ES, Koester A, Paulsen AQ et al (2002) Mimecan/osteoglycin-deficient mice have collagen fibril abnormalities. Mol Vis $8: 407-415$

33. Tasheva ES, Ke A, Deng Y et al (2004) Differentially expressed genes in the lens of mimecan-null mice. Mol Vis 10:403-416 